\title{
Ellipticity Dependence of Excitation and Ionization of Argon Atoms by Short-Pulse Infrared Radiation
}

\author{
Thomas Pauly ${ }^{1}$, Aaron Bondy ${ }^{1,2}$, Kathryn R. Hamilton ${ }^{1}$, \\ Nicolas Douguet ${ }^{3}$, Xiao-Min Tong ${ }^{4}$, Dashavir Chetty ${ }^{5}$, and K. Bartschat ${ }^{1}$ \\ ${ }^{1}$ Department of Physics and Astronomy, Drake University, Des Moines, Iowa 50311, USA \\ ${ }^{2}$ Department of Physics, University of Windsor, Windsor, Ontario N9B 3P4, Canada \\ ${ }^{3}$ Department of Physics, Kennesaw State University, Kennesaw, Georgia 30144, USA \\ ${ }^{4}$ Center for Computational Sciences, University of Tsukuba, \\ 1-1-1 Tennodai, Tsukuba, Ibaraki 305-8573, Japan and \\ ${ }^{5}$ Centre for Quantum Dynamics, Griffith University, Brisbane, Queensland 4111, Australia
}

(Dated: July 2, 2020)

\begin{abstract}
When atoms or molecules are exposed to strong short-pulse infrared radiation, ionization as well as "frustrated tunneling ionization" (FTI) can occur, in which some of the nearly freed electrons recombine into the initial ground or an excited bound state. We analyze the ellipticity dependence of the relative signals that are predicted in a single-active electron approximation ( $\mathrm{SAE}$ ), the validity of which is checked against a parameter-free multi-electron $R$-matrix (close-coupling) with time dependence approach. We find good agreement between the results from both models, thereby providing confidence in the SAE model potential to treat the process of interest. Comparison of the relative excitation probabilities found in our numerical calculations with the predictions of Landsman et al. (New Journal of Physics 15 (2013) 013001) and Zhao et al. (Optics Express 27 (2019) 21689) reveals good agreement with the former for short pulses. For longer pulses, the ellipticity dependence becomes wider than that obtained from the Landsman et al. formula, but we do not obtain the increase compared to linearly polarized radiation predicted by Zhao et al.
\end{abstract}

\section{INTRODUCTION}

Strong-field ionization utilizes a slowly varying fewcycle infrared laser pulse to eject an electron from an atom. With an intense infrared laser, the electric field significantly alters the effective potential. In this process described by Corkum [1], the electron has the ability to "tunnel" through the effective barrier and become free. After ionization, however, the electric field can guide the electron back to the target ion. There it can be recaptured into the ground state or any other bound state, such as high-lying Rydberg states. This is the strong-field process of "frustrated tunnel ionization" (FTI), which is negligible in the treatment of the standard photoeffect. From some of these excited bound states, the electron can jump to metastable states of the atom. Production of metastable sources through FTI may have practical advantages, for example, a potentially high yield with minimal heating of the sample [2].

In addition to studying the process with linearly polarized light, the ellipticity dependence of both FTI and successful ionization has been of significant theoretical interest. Intuitively, one might expect that FTI will diminish with increasing ellipticity of the IR radiation, since rescattering is most likely a very important contributing process, in addition to direct excitation. Successful ionization, on the other hand, while also potentially benefitting from recollisions, may be less sensitive to the latter. If these hypotheses are correct, one would expect the rates for these processes, at a fixed peak intensity of a pulse (in practice, a fixed total energy delivered by the pulse), to peak at or near zero ellipticities, i.e., linearly or (near-)linearly polarized light. It should then drop off with increasing ellipticity and have a minimum for (near-)circularly polarized light. Also, the drop should be steeper for FTI than for actual ionization, as discussed in [3].

An early FTI experiment was performed by Nubbemeyer et al. [4] on helium, where the above expectations were, indeed, fulfilled. Since FTI is a strong-field process, in which the motion of the electron is predominantly driven by the slowly varying electric field, it is not surprising that models were developed in which the motion of the electron was described classically, with very limited account for the target structure. Landsman et al. [5], for example, derived an analytical formula based on the strong-field approximation model, which neglected the effect of the Coulomb field until the pulse has passed. They considered the conditions where the initial transverse momentum of the tunneled electron is cancelled by the drift momentum gained in the field. This results in a Gaussian probability distribution with respect to ellipticity, which depends only on the laser intensity, wavelength, and ionization potential of the target. The approximations employed in the derivation of the formula limit it, in principle, to laser fields much stronger than the Coulomb field and small $\varepsilon$, but it was apparently sufficient to reproduce the experimental results of Nubbemeyer et al. [4]. More recent work on the helium target was reported by Yun et al. [6].

For situations involving targets with lower ionization potentials or lower laser intensities, semi-classical calculations that account for the Coulomb field even during the laser interaction, such as those presented by ShvetsovShilovski et al. [7], are typically employed. At lower intensities, these calculations sometimes predict a max- 
imum in the excitation probability at a nonzero ellipticity. Zhao et al. [8], for example, recently reported predictions for FTI of $\operatorname{Ar}(3 p)$ with laser pulses of two wavelengths $(800 \mathrm{~nm}$ and $1,600 \mathrm{~nm})$, two peak intensities $\left(0.8 \times 10^{14} \mathrm{~W} / \mathrm{cm}^{2}\right.$ and $1.6 \times 10^{14} \mathrm{~W} / \mathrm{cm}^{2}$, and pulselengths of 30,10 , and 4 cycles, respectively. At the higher intensity, the distribution deviates from a Gaussian shape, exhibiting a relatively flat top around linear polarization $\varepsilon=0.0$. At the lower intensity and a pulse length of $30 \mathrm{fs}$ for the $800 \mathrm{~nm}$ radiation, a maximum of the relative FTI rate occurs at $\varepsilon=0.2$, where there is a predicted increase of more than $10 \%$ compared to $\varepsilon=0$. For shorter pulses of 10 and 4 cycles, the increase disappears in the Zhao et al. [8] predictions. We note that peaks at $\varepsilon \neq 0$ were previously predicted for $\mathrm{Mg}[9]$, where it was shown that they are sensitive to the initial conditions of the calculations.

No experimental data to confirm or disprove the Zhao et al. [8] predictions are currently available, but an experiment is underway at Griffith University. Preliminary results were already announced [10], but no firm conclusions are available yet. In fact, further theoretical guidance is highly desirable for this challenging experimental project.

Very recently, experimental FTI and ionization results for $\operatorname{Ar}(3 p)$ obtained with linear polarization were published [11]. These data were compared with calculations carried out by the authors of the present paper. Good qualitative agreement was obtained with predictions from a single-active-electron (SAE) model, with the effective potential proposed by Tong and Lin [12]. A few calculations to cross-check those results were also carried out with other potentials proposed in the literature, and most importantly - with predictions from a fully $a b i n i-$ tio all-electron $R$-matrix with time dependence (RMT) approach. The latter (see below) is based on the closecoupling expansion, with full account for electron exchange where it is potentially important and a multiconfiguration Hartree-Fock target description.

The principal objectives of the present work were to extend those latter calculations to elliptically polarized light. This would allow us to i) further test the validity of the SAE approach by comparing with RMT predictions, ii) once again check the predictions of Landsman et al. [5] and Zhao et al. [8] (which clearly disagree for the $800 \mathrm{~nm}, 30 \mathrm{fs}, 0.8 \times 10^{14} \mathrm{~W} / \mathrm{cm}^{2}$ case), and iii) provide guidance for the experimental investigations. In the latter context, it is also of interest to determine how the experimental signal might actually arise. The measured observable is the relative yield of metastable Ar atoms in the $\left(3 p^{5} 4 s\right)^{3} P_{2}$ and $\left(3 p^{5} 4 s\right)^{3} P_{0}$ states. Given that the ground state is a relatively well $L S$-coupled singlet state, while the metastables are equally well $L S$-coupled triplet states, the process almost certainly proceeds via intermediately-coupled excited states, whose triplet component can be reached while fulfilling the spin-conserving selection rule of electric dipole radiation.

To model the actual experiment, therefore, one would ideally carry out a semi-relativistic calculation accounting for the intermediate-coupling nature of the target states involved, followed by a set of rate equations to predict the ultimate fate of the $3 p$ electron that is subjected to the strong laser field. Even though the RMT method has recently been extended to the semirelativistic regime [13], such calculations for the process of interest here are far beyond the currently available computational resources. Fortunately, as shown in [11], the relative probabilities as a function of laser parameters appear to be predictable without these efforts, and hence the present calculations are expected to be useful in the further planning of the experimental study.

This paper is organized as follows. In section II we briefly describe the SAE and RMT models used in the present calculations. This is followed by our results presented in section III, with the conclusions drawn in section IV. Unless specified otherwise, atomic units are used throughout.

\section{COMPUTATIONAL DETAILS}

\section{A. Single-Active-Electron Approach}

The basic principles behind these calculations were described in [11]. In modeling the process, we need to solve the nonrelativistic time-dependent Schrödinger equation (TDSE). In atomic units, this is a partial differential equation

$$
\mathrm{i} \frac{\partial}{\partial t} \Psi(\boldsymbol{r}, t)=\left[H_{0}+V(t)\right] \Psi(\boldsymbol{r}, t)
$$

for the wave function $\Psi(\boldsymbol{r}, t)$. Here $H_{0}$ is the field-free Hamiltonian and $V(t)$ is the time-dependent interaction represented by the laser pulse. Instead of including the kinetic energy and the Coulomb interaction of all electrons with the nucleus and with each other, only one electron is considered in the Single-Active-Electron (SAE) approximation. Its interaction with the nucleus and all other electrons is approximated by a (usually local) potential. For the present work, we used the potential proposed by Tong and Lin [12]), which was recently employed successfully in FTI calculations with linearly polarized radiation [11]. For maximum internal consistency, we recalculated the $3 p$ and all other orbitals in this potential, and we also used it to generate the distorted Coulomb waves needed to extract the ionization probability.

The electric field of the laser is represented by the vector potential

$$
\boldsymbol{A}(t)=f(t) \frac{E_{0}}{\omega \sqrt{1+\varepsilon^{2}}}[\cos (\omega t+\varphi) \hat{\boldsymbol{x}}+\varepsilon \sin (\omega t+\varphi) \hat{\boldsymbol{y}}] .
$$

Here $\varepsilon$ is the ellipticity, with $\varepsilon=0$ corresponding to linearly polarized and $\varepsilon=1$ to circularly polarized light. 
Furthermore, $E_{0}$ is the maximum amplitude of the electric field, and $\varphi$ is the carrier-envelope phase (CEP). The factor $E_{0} / \sqrt{1+\varepsilon^{2}}$ ensures that for a given pulse represented by the envelope function $f(t)$ (typically a Gaussian or a $\sin ^{2}$ function closely resembling a Gaussian), the same amount of energy (i.e., the field squared integrated over the length of the pulse) is delivered to the target. This is needed to unambiguously test the effects of different ellipticities. In order to avoid a potentially unphysical displacement [14], we set the vector potential and calculate the electric field as its time derivative according to $\boldsymbol{E}(t)=-\frac{d}{d t} \boldsymbol{A}(t)$.

In writing Eq. (2), we chose the quantization axis $(\hat{\boldsymbol{z}})$ perpendicular to the electric field, i.e., along the laser propagation direction. For the special case of linear polarization $(\varepsilon=0)$, it is better to rotate the coordinate system and take advantage of the cylindrical symmetry by choosing the quantization axis parallel to the direction of the electric field vector.

Since the initial state is spherically symmetric, rightand left-hand elliptically polarized light yield the same answers. In the electric dipole approximation, there is no spatial dependence in $\boldsymbol{A}(t)$ and $\boldsymbol{E}(t)$. We use $\sin ^{2}$ envelopes with FWHM of the intensity set to 6 fs or $30 \mathrm{fs}$, respectively. For $800 \mathrm{~nm}$, the FWHM time in fs is very close to the number of cycles for a $\sin ^{2}$ envelope of the field. In the 6 fs calculations, we average over the CEP by performing calculations for $\varphi=0^{\circ}, 45^{\circ}, 90^{\circ}$ and $135^{\circ}$.

The initial state of the system (a $3 p$ orbital with a magnetic quantum number $m$ ) is propagated in time until the end of the pulse. In order to simulate an unpolarized $3 p$ electron in the Ar atom, calculations are carried out for all possible initial projections of the orbital angular momentum, i.e., $m=0, \pm 1$, and the results are then summed incoherently. For linearly polarized radiation, the results for initial $m=+1$ and $m=-1$ are the same and the value of $m$ is conserved. As mentioned above, we then choose the quantization axis along the direction of the electric field. In this case, we use the straightforward Crank-Nicolson (CN) method [15] with the electric dipole operator written in the length gauge to propagate the wave function.

The case of a nonzero ellipticity, on the other hand, is much more numerically challenging due to the reduced symmetry that no longer conserves the initial projection of the angular momentum. Here we choose the quantization axis perpendicular to the electric field, i.e., along the laser propagation direction, as written in Eq. (2). Since the CN method is no longer applicable directly, we employ the matrix iterative method (MIM) [16] with the dipole operator chosen in the velocity gauge.

After the pulse is over, the wave function of the active electron is projected onto the initial $3 p$ orbital to obtain the survival probability $P_{\text {surv }}$ as the square of the overlap matrix element, as well as onto selected excited states to yield individual excitation probabilities and finally on distorted Coulomb waves to obtain the energy-differential ionization probability $d P / d E$, where
$E$ is the energy of the continuum electron. The integral over all ejected energies yields the total ionization probability $P_{\text {ion }}$. Finally, the total probability for excitation $P_{\text {exc }}$ (regardless of the Rydberg state) is obtained by using the conservation of the norm of the total wave function, i.e., $P_{\mathrm{exc}}=1-P_{\mathrm{surv}}-P_{\mathrm{ion}}$. Special care was taken to ensure that the numerics were sufficiently stable to ensure meaningful results, even when $P_{\text {surv }}$ and $P_{\text {ion }}$ are small.

All the SAE calculations reported below were carried out with an updated version of the computer code described by Douguet et al. [17] and references therein. Also, spot checks against results from the independent SAE code used for most of the calculations reported in [11] were performed.

\section{B. R-matrix with Time Dependence Approach}

The $R$-matrix with time-dependence method (RMT) is an ab initio, multi-electron method capable of describing the interaction of general atoms and molecules with arbitrary laser fields [18-20]. RMT employs the wellknown $R$-matrix paradigm [21] of dividing configuration space into two separate regions, in this case over the radial coordinate of the ejected electron. In the inner region (close to the nucleus) the time-dependent multielectron wave function is represented by an $R$-matrix basis with time-dependent coefficients. This configurationinteraction approach ensures that we take into full account all electronic interactions, including electron correlation and exchange. Specifically, we used the target description developed by Burke and Taylor [22] for photoionization of argon.

In the outer region, where one electron is sufficiently far removed that we can neglect the effect of exchange, the wave function is described in terms of residual-ion states coupled with the radial wave function of the ejected electron, and is expressed explicitly on a finite-difference grid. The outer region also includes several long-range potentials, which describe the interactions between the ejected electron, laser and residual ion.

In contrast to traditional $R$-matrix-based approaches, the wave function itself is matched explicitly at the boundary, in both directions, rather than via an $R$ matrix. The wave function is propagated in the length gauge, as it has been found to converge faster than the velocity gauge with the atomic structure description typically employed in time-dependent $R$-matrix calculations [23]. Time propagation of the wave function is achieved by the Krylov subspace method of Arnoldi and Lanczos [24]. RMT has been successfully applied to a wide range of strong-field problems, from High Harmonic Generation (HHG) in two-color [25] and near-IR fields [26], XUV-initiated HHG [27, 28], and strong-field rescattering [29].

The default polarization plane in the RMT code is the $z y$-plane, with $\hat{\boldsymbol{z}}$ and $-\hat{\boldsymbol{y}}$ the major and minor axes of the 
polarization ellipse, respectively. A general, elliptically polarized electric field will therefore be of the form:

$$
\boldsymbol{E}(t)=f(t) \operatorname{Re}\left[\hat{\boldsymbol{e}} \mathrm{e}^{-\mathrm{i}(\omega t+\varphi)}\right]
$$

where $\hat{\boldsymbol{e}}=(\hat{\boldsymbol{z}}-\mathrm{i} \varepsilon \hat{\boldsymbol{y}}) / \sqrt{1+\varepsilon^{2}}$ is an arbitrary polarization vector and $\operatorname{Re}[x]$ denotes the real part of the complex quantity $x$. As before, $\varepsilon$ is the ellipticity and $f(t)$ is the envelope function, chosen to create a $\sin ^{2}$ pulse envelope, and $\varphi$ is a carrier-envelope phase. This choice of polarization axes allows calculations for linear fields $(\varepsilon=0)$ to be polarized along the $z$-axis. For calculations with circularly polarized laser pulses, it is often practical to rotate the polarization plane to the $x y$-plane, as this reduces the number of dipole-accessible states relative to the $z y$-plane, thereby reducing the size of the calculation significantly.

In the current study, the argon target is described within an $R$-matrix inner region of radius 20 and an outer region of 3,500 . The latter ensures that no significant part the wave packet describing the ejected electron gets to the boundary, where it would be reflected. The finite-difference grid spacing in the outer region is 0.08 and the time step for the wave function propagation is 0.01 ( $=0.24$ attoseconds). The description of argon includes all available $3 s^{2} 3 p^{5} \epsilon \ell$ and $3 s 3 p^{6} \epsilon \ell$ channels up to a maximum total angular momentum of $L_{\max }=80$. Doubly-excited states of the residual $\mathrm{Ar}^{+}$ion are also included to ensure an accurate description of the $3 s 3 p^{6} n p$ window resonances [30].

The continuum functions are constructed from a set of $50 \mathrm{~B}$-splines, of order 13 , for each available angular momentum of the outgoing electron. The ground-state survival probability is determined directly from the wave function at the end of the laser pulse. The ionization probability is calculated by firstly projecting the channelresolved wave functions, from some radius $r_{\text {min }} \geq 20$ up to $r_{\max }=3,500$, onto plane waves to determine the photoelectron spectrum, and then integrating over all energies. As with the SAE method outlined above, the total excitation probability is then obtained using the conservation of the norm of the wave function.

Due to the significant computational expense of these calculations, RMT results are only available for select ellipticities at a laser intensity of $1.0 \times 10^{14} \mathrm{~W} / \mathrm{cm}^{2}$. In principle, the coupled-channel formulation used here also allows ionization leaving the residual $\mathrm{Ar}$ ion in the $\left(3 s 3 p^{6}\right)^{2} S$ state. However, this excited ionic state has a much higher ionization potential, and hence the signal for the process of interest here is negligible.

\section{RESULTS AND DISCUSSION}

Figure 1 depicts a comparison of SAE results for three different CEPs of a 6 -cycle $800 \mathrm{~nm}$ pulse of peak intensity $10^{14} \mathrm{~W} / \mathrm{cm}^{2}$. Also shown are results from our RMT

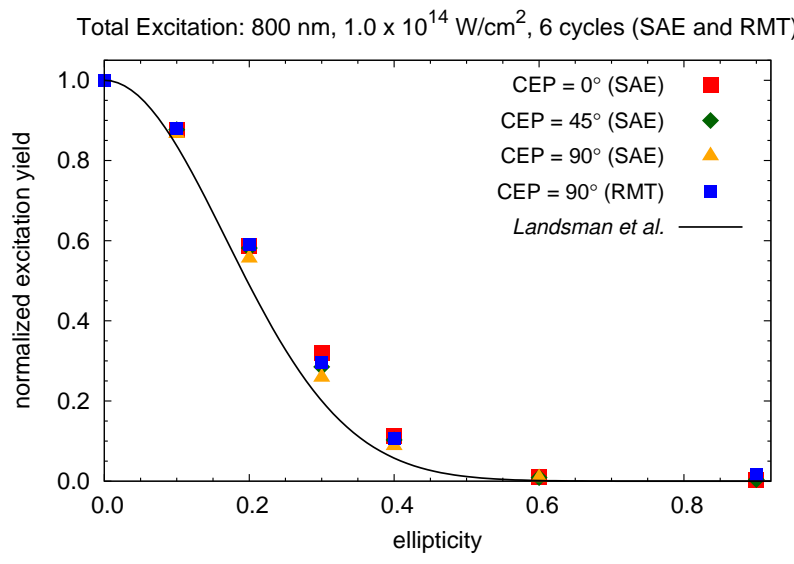

FIG. 1: Present SAE results for total excitation of $\operatorname{Ar}(3 p)$ for a 6 -cycle $800 \mathrm{~nm}$ pulse of peak intensity $10^{14} \mathrm{~W} / \mathrm{cm}^{2}$ for three different CEPs, compared with the predictions obtained from the Landsman et al. formula and an RMT calculation for a CEP of $90^{\circ}$. See text for details.

calculation and the predictions obtained from the Landsman et al. formula. Recall that the electric field rather than the vector potential is set in the RMT code, and so the RMT calculation was carried out for an electric field with $\mathrm{CEP}=0^{\circ}$, which is equivalent to an SAE calculation with $\mathrm{CEP}=90^{\circ}$, assuming that the derivative of the envelope function only has a minor effect on the calculation of the field used in the SAE calculation. To simplify the comparison, we therefore use the label $90^{\circ}$ for the RMT calculations. We also checked that SAE calculations performed with setting the electric field directly (i.e., as in RMT), while producing an nonzero displacement and small changes in the individual probabilities, hardly affect the ratios and hence the conclusions of the present work. Clearly, all the results are so close to each other that the all-electron RMT results for the observable of interest can certainly be taken as support for the much simpler SAE calculations.

Generally, our results for the 6-cycle pulses are in qualitative agreement with the Landsman et al. formula, even though they assumed "long" pulses in their derivation and made further approximations mentioned in the Introduction. However, we note a small but systematic trend of the numerical results always lying slightly above those predicted by the formula. Finally, averaging over the CEP only has a small effect on the 6-cycle results.

Figure 2 shows a comparison between the present SAE results and the predictions of Landsman et al. [5] and Zhao et al. [8] for a central wavelength of $800 \mathrm{~nm}$ and several peak intensities and pulse lengths. Most of our calculations were performed for 6-cycle pulses and averaged over four CEPs, as described above. The formula given in [5] contains neither the pulse length nor the CEP. The simulations in [8] were performed for 30-cycle, 10-cycle, and 4-cycle pulses. The most interesting results appears to be the one for 30 cycles, $800 \mathrm{~nm}$, and a peak intensity of $0.8 \times 10^{14} \mathrm{~W} / \mathrm{cm}^{2}$, where the relative FTI maximum is 


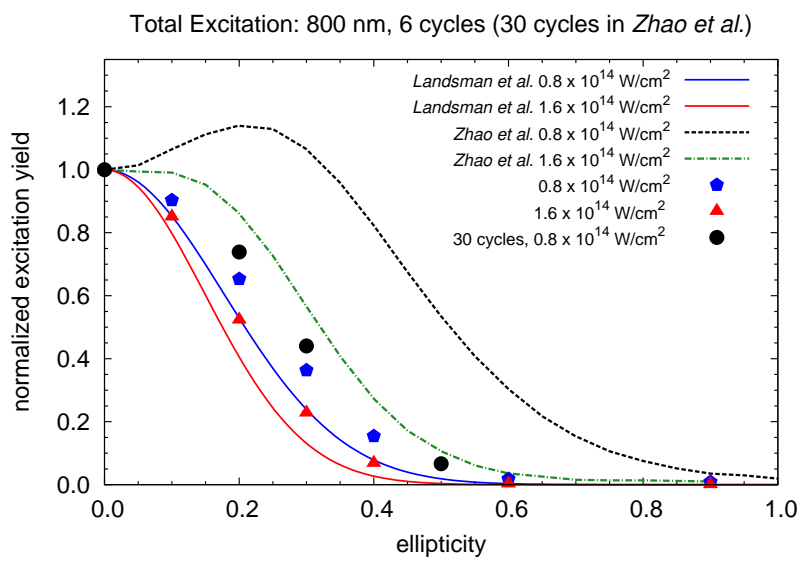

FIG. 2: Comparison of the present SAE results for total excitation with the predictions obtained from the Landsman et al. formula and those of Zhao et al. for a central wavelength of $800 \mathrm{~nm}$ and peak intensities of $0.8 \times 10^{14} \mathrm{~W} / \mathrm{cm}^{2}$ and $1.6 \times 10^{14} \mathrm{~W} / \mathrm{cm}^{2}$. See text for details.

predicted to have an unambiguous maximum at $\varepsilon \approx 0.2$. In order to check this, we carried out several calculations for 30 cycles, so that it was possible to obtain the relevant ratios. The calculations with a small but nonzero ellipticity such as $\varepsilon=0.2$ are computationally demanding, and hence we do not map out the entire curve. However, the available points at $\varepsilon=0.2,0.3$, and 0.5 clearly show that the width of the curve representing the ratio of the excited-state population normalized to its value at $\varepsilon=0.0$ is significantly wider for the 30 fs pulse compared to the 6 fs pulse at $0.8 \times 10^{14} \mathrm{~W} / \mathrm{cm}^{2}$, albeit not by as much as predicted by Zhao et al. [8]. We still find the maximum at $\varepsilon=0.0$.

The apparently better agreement of our calculations for $1.6 \times 10^{14} \mathrm{~W} / \mathrm{cm}^{2}$ and the Landsman et al. formula for $0.8 \times 10^{14} \mathrm{~W} / \mathrm{cm}^{2}$ is accidental. It simply reflects the fact that the Landsman et al. formula predicts the curve too narrow. Similarly, the better agreement with Zhao et al. also for the higher intensity is due to their method predicting the curve too wide. This clearly demonstrates that for the processes discussed in the present paper neither one of the essentially classical methods is sufficiently accurate. Instead a quantum-mechanical treatment is highly desirable.

Figure 3 shows results for yet another peak intensity, this time $2 \times 10^{14} \mathrm{~W} / \mathrm{cm}^{2}$. Here we investigate the ellipticity dependence of ending up in the $4 s$ orbital, any of the $n=5$ or $n=6$ orbitals, or any excited state directly at the end of the laser pulse. Interestingly, the $4 s$ excitation is predicted to peak at a nonzero ellipticity while $n=5$ and $n=6$ are very similar to the entire relative excitation probability. Recall that the experimentally observed signal originates from the metastable states involving the $4 s$ orbital. However, this is not the signal that one would expect from the target immediately after the laser pulse is over but rather much later (on an atomic timescale) when exited states have optically de-
Excitation: $800 \mathrm{~nm}, 2 \times 10^{14} \mathrm{~W} / \mathrm{cm}^{2}, 6$ cycles, CEP avg. (SAE)

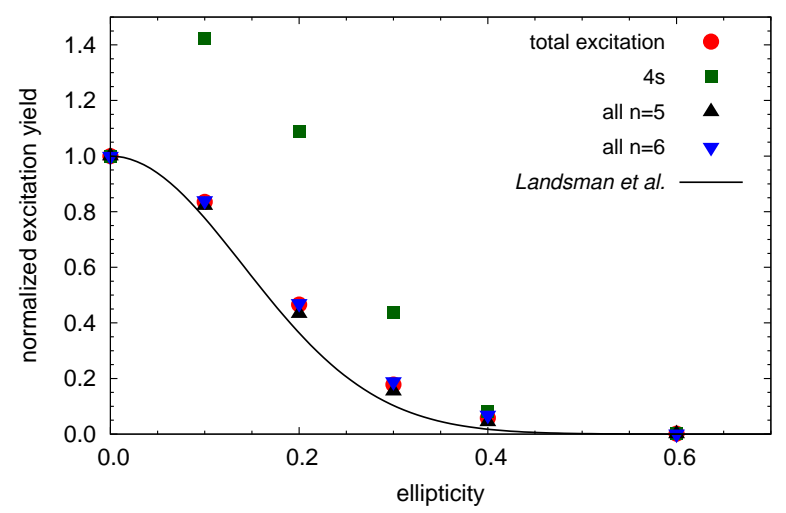

FIG. 3: CEP-averaged SAE results for total excitation of $\operatorname{Ar}(3 p)$ for a 6 -cycle $800 \mathrm{~nm}$ pulse of peak intensity $2 \times$ $10^{14} \mathrm{~W} / \mathrm{cm}^{2}$. We present the relative ellipticity dependence for ending up in the $4 s$ orbital as well as all the $n=5$ and $n=6$ orbitals, respectively. Also shown are the predictions from the Landsman et al. formula. See text for details.

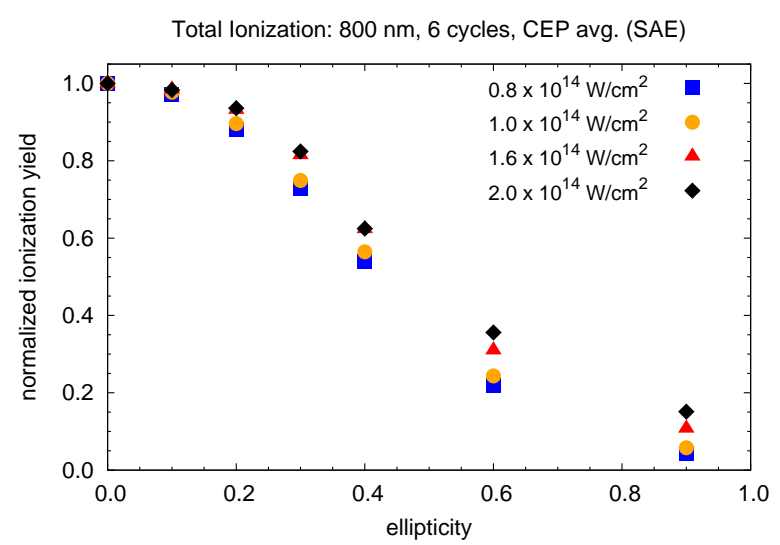

Total lonization: $800 \mathrm{~nm}, 1.0 \times 10^{14} \mathrm{~W} / \mathrm{cm}^{2}, 6$ cycles (SAE and RMT)

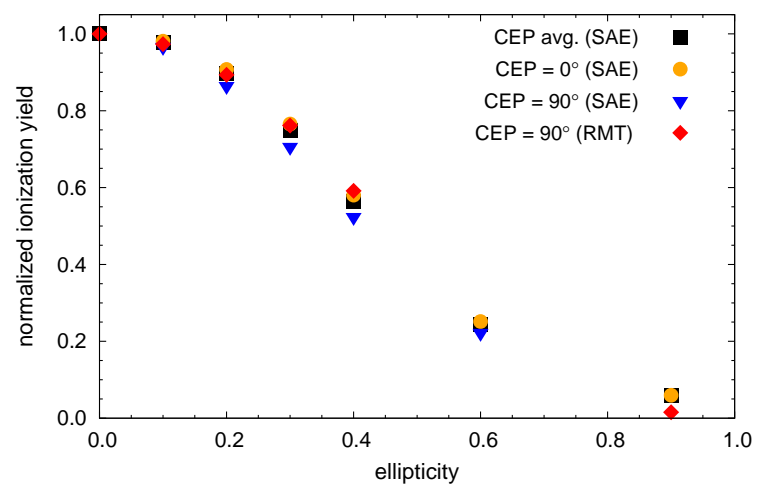

FIG. 4: Top: CEP-averaged SAE results for the relative ellipticity dependence of $\operatorname{Ar}(3 p)$ ionization for a 6 -cycle $800 \mathrm{~nm}$ pulse of various peak intensities indicated in the legend. Bottom: Comparison of SAE results for ionization for a 6-cycle $800 \mathrm{~nm}$ pulse of peak intensity $10^{14} \mathrm{~W} / \mathrm{cm}^{2}$ compared with RMT results for a few ellipticities. 
cayed either back to the ground state or to either one of the $\left(3 p^{5} 4 s\right)^{3} P_{2,0}$ metastable states.

Finally, we show in Fig. 4 the relative ionization probability for a number of peak intensities of a 6-cycle $800 \mathrm{~nm}$ pulse obtained in the SAE model (top panel) as well as for a peak intensity of $10^{14} \mathrm{~W} / \mathrm{cm}^{2}$ for two CEPs, CEPaveraged, and compared once again with RMT results for a CEP of $90^{\circ}$. Compared to the previous figures, we clearly see a much wider curve than for FTI. Even for $\varepsilon=0.9$, the signal can visually be distinguished from zero. Not surprisingly in light of the previous results, the CEP dependence is weak even for a short 6-cycle pulse. Also, the RMT calculations once again reveal a similar ellipticity dependence as predicted by the SAE model.

\section{CONCLUSIONS}

We have investigated the ellipticity dependence of frustrated and completed ionization of the $\operatorname{Ar}\left(3 p^{6}\right)$ ground state, when the target was exposed to a 6-cycle or in some cases 30-cycle $800 \mathrm{~nm}$ infrared pulse of with peak intensities between $0.8 \times 10^{14} \mathrm{~W} / \mathrm{cm}^{2}$ and $2.0 \times 10^{14} \mathrm{~W} / \mathrm{cm}^{2}$, respectively. Predictions based on calculations in the single-active electron approximation were spot-checked against results obtained in a multi-electron $R$-matrix (close-coupling) with time dependence model. Excellent agreement was obtained for the ratio of these probabilities as a function of ellipticity when normalized to the results for linearly polarized light. In practice, this ratio is close, although not identical, to what would be measured experimentally.

Reasonable agreement with the analytic formula pro- posed by Landsman et al. [5] for the relative ellipticity was obtained for the 6-cycle pulses. For the longer 30cycle pulse with a peak intensity of $0.8 \times 10^{14} \mathrm{~W} / \mathrm{cm}^{2}$, the curve widens. This is in qualitative agreement with the predictions of Zhao et al. [8], but we do not confirm an increase at nonzero ellipticity. In general, the Landsman et al. formula appears to predict the curve too narrow while the treatment of Zhao et al. predicts it too wide. Finally, we confirm that the relative ellipticity dependence for ionization follows a much wider bell curve than that for frustrated ionization.

\section{Acknowledgments}

This work was supported by the United States National Science Foundation under grants No. OAC1834740 and PHY-1803844, and by the XSEDE supercomputer allocation No. PHY-090031. The calculations were carried out on Stampede2 and Frontera at the Texas Advanced Computing Center, as well as Bridges at the Pittsburgh Supercomputer Center and Comet at the San Diego Supercomputer Center. A.B. is grateful for a Michael Smith Scholarship. X.-M.T. was supported by a Grant-in-Aid for Scientific Research (Grant No. JP16K05495) from the Japan Society for the Promotion of Science. D.C. is supported by an Australian Government RTP Scholarship. The RMT code is part of the UK-AMOR suite. It can be obtained free of charge from https://gitlab.com/uk-amor/rmt/.
[1] P. B. Corkum, Phys. Rev. Lett. 71, 1994 (1993).

[2] M. Dakka, G. Tsiminis, R. Glover, C. Perrella, J. Moffatt, N. Spooner, R. Sang, P. Light, and A. Luiten, Physical Review Letters 121 (2018).

[3] X. Gao and X.-M. Tong, Phys. Rev. A 100, 063424 (2019).

[4] T. Nubbemeyer, K. Gorling, A. Saenz, U. Eichmann, and W. Sandner, Phys. Rev. Lett. 101, 233001 (2008).

[5] A. S. Landsman, A. N. Pfeiffer, C. Hofmann, M. Smolarski, C. Cirelli, and U. Keller, New Journal of Physics 15, 013001 (2013).

[6] H. Yun, J. H. Mun, S. B. Park, I. A. Ivanov, C. H. Nam, and K. T. Kim, Nature Photonics 12, 620 (2018).

[7] N. I. Shvetsov-Shilovski, S. P. Goreslavski, S. V. Popruzhenko, and W. Becker, Laser Physics 19, 1550 (2009).

[8] Y. Zhao, Y. Zhou, J. Liang, Z. Zeng, Q. Ke, Y. Liu, M. Li, and P. Lu, Opt. Express 27, 21689 (2019).

[9] K.-y. Huang, Q.-z. Xia, and L.-B. Fu, Phys. Rev. A 87, $033415(2013)$.

[10] D. Chetty, R. D. Glover, H. Xu, A. J. Palmer, B. A. deHarak, P. S. Light, A. N. Luiten, I. V. Litvinyuk, and R. T. Sang, in Book of Abstracts: XXXI International
Conference on Photonic, Electronic, and Atomic Collisions (ICPEAC, 2019), p. 81.

[11] D. Chetty, R. D. Glover, B. A. deHarak, X. M. Tong, H. Xu, T. Pauly, N. Smith, K. R. Hamilton, K. Bartschat, J. P. Ziegel, et al., Phys. Rev. A 101, 053402 (2020).

[12] X. M. Tong and C. D. Lin, J. Phys. B: At. Mol. Opt. Phys. 38, 2593 (2005).

[13] J. Wragg, D. D. A. Clarke, G. S. J. Armstrong, A. C. Brown, C. P. Ballance, and H. W. van der Hart, Phys. Rev. Lett. 123, 163001 (2019).

[14] I. A. Ivanov, A. S. Kheifets, K. Bartschat, J. Emmons, S. M. Buczek, E. V. Gryzlova, and A. N. GrumGrzhimailo, Phys. Rev. A 90, 043401 (2014).

[15] J. Crank and P. Nicolson, Advances in Computational Mathematics 6, 207 (1996).

[16] M. Nurhuda and F. H. M. Faisal, Phys. Rev. A 60, 3125 (1999).

[17] N. Douguet, A. N. Grum-Grzhimailo, E. V. Gryzlova, E. I. Staroselskaya, J. Venzke, and K. Bartschat, Phys. Rev. A 93, 033402 (2016).

[18] A. C. Brown, G. S. Armstrong, J. Benda, D. D. Clarke, J. Wragg, K. R. Hamilton, Z. Man, J. D. Gorfinkiel, and H. W. van der Hart, Computer Physics Communications 
250, 107062 (2020), ISSN 0010-4655.

[19] D. D. A. Clarke, G. S. J. Armstrong, A. C. Brown, and H. W. van der Hart, Phys. Rev. A 98, 053442 (2018).

[20] L. R. Moore, M. A. Lysaght, L. A. A. Nikolopoulos, J. S. Parker, H. W. van der Hart, and K. T. Taylor, J. Mod. Optics 58, 1132 (2011).

[21] P. G. Burke, R-matrix Theory of Atomic Collisions (Springer Berlin Heidelberg, 2011).

[22] P. G. Burke and K. T. Taylor, J. Phys. B. At. Mol. Opt. Phys. 8, 2620 (1975).

[23] S. Hutchinson, M. A. Lysaght, and H. W. van der Hart, J. Phys. B. At. Mol. Opt. Phys. 43, 095603 (2010).

[24] W. E. Arnoldi, The Quarterly Journal of Pure and Applied Mathematics 9, 17 (1951).
[25] K. R. Hamilton, H. W. van der Hart, and A. C. Brown, Phys. Rev. A 95, 013408 (2017).

[26] O. Hassouneh, A. C. Brown, and H. W. van der Hart, Phys. Rev. A 90, 043418 (2014).

[27] A. C. Brown and H. W. van der Hart, Phys. Rev. Lett. 117, 093201 (2016).

[28] D. D. A. Clarke, H. W. van der Hart, and A. C. Brown, Phys. Rev. A 97, 023413 (2018).

[29] O. Hassouneh, S. Law, S. F. C. Shearer, A. C. Brown, and H. W. van der Hart, Phys. Rev. A 91, 031404 (2015).

[30] M. Levy and R. Huffman, Journal of Quantitative Spectroscopy and Radiative Transfer 9, 1349 (1969). 